\title{
Freeze-fracture Electron Microscopy on Lipid-stabilized Gas Bubbles
}

\author{
B. Papahadjopoulos-Sternberg and J. Ackrell
}

NanoAnalytical Laboratory, 3951 Sacramento Street, San Francisco, CA 94118, USA

Suspensions of lipid-stabilized microbubbles are frequently used for biomedical applications such as ultrasound contrast enhancement, ultrasound-promoted drug and gene delivery, and blood substitution [1]. The potency of such microbubbles is strongly depending upon their morphology adopted in a biological relevant environment. Freeze-fracture electron microscopy is not only a powerful technique to characterize such micrometer-size drug/gene carriers but also the method of choice to study their fate related to drug/gene load, application milieu, and during interaction with cells. Furthermore it allows distinguishing between bilayer and non-bilayer structures [2-8].

Using freeze-fracture electron microscopy we studied the morphology of a commercially available, product (Definity ${ }^{\circledR} /$ Bristol-Myers Squibb Medical Imaging, Inc.) for ultrasound contrast enhancement after intravenous application.

As visible from Fig. 1A the non-activated sample contains small, mostly spherical particles showing diameters in the size range of 30 to $70 \mathrm{~nm}$. These particles display their shadows behind their structures what is characteristic for "hard-core" particles such as micelles. Because of their larger size than "normal" micelles we assume they represent mixed micelles. The very same samples, activated by a high-frequent, short time impulse, display large gas bubbles (Fig. 1B). They show diameters between 200 to $5000 \mathrm{~nm}$ what is about 50 times larger than the starting micelles. Similar, but smaller and more stressed gas bubbles are visible in the activated, but 250 times diluted sample (Fig. 1C). Most of the lipid-stabilized gas bubbles display their shadows in front of their structures what represents the proof that these gas bubbles are coated by a lipid monolayer. In this monolayer the hydrophilic lipid headgroups are reaching into the water phase while their hydrophobic tails are directed into the gas phase. Contrary to lipid-monolayer stabilized gas bubbles bilayer vesicles such as liposomes show concave and convex fracture planes (shadow in front and behind their structures respectively, Fig. 2B). In order to obtain thicker lipid monolayer stabilizing the gas bubbles oil was added. In such preparations oil domains are observed within the lipid monolayer displaying diameters of about $200 \mathrm{~nm}$ and highs of $2 \mathrm{~nm}$ (Fig. 2A).

\section{References}

[1] J. R. Lindner Nature Rev. Drug Discovery 3 (2004) 527.

[2] B. Sternberg, Liposome Technology, CRC Press I (1992) 363.

[3] B. Sternberg, Handbook Nonmedical Applications of Liposomes CRC Press (1996) 271.

[4] B. Sternberg, Medical Applications of Liposomes, Elsevier (1998) 395.

[5] B. Sternberg et al., Nature 378 (1995) 21.

[6] V. P. Torchilin et al. PNAS (2003) 100 (4) 1972.

[7] V. P. Torchilin et al. PNAS (2003) 100 (10) 603.

[8] P. C. Bell et al., accepted by Biochemistry (2006) 

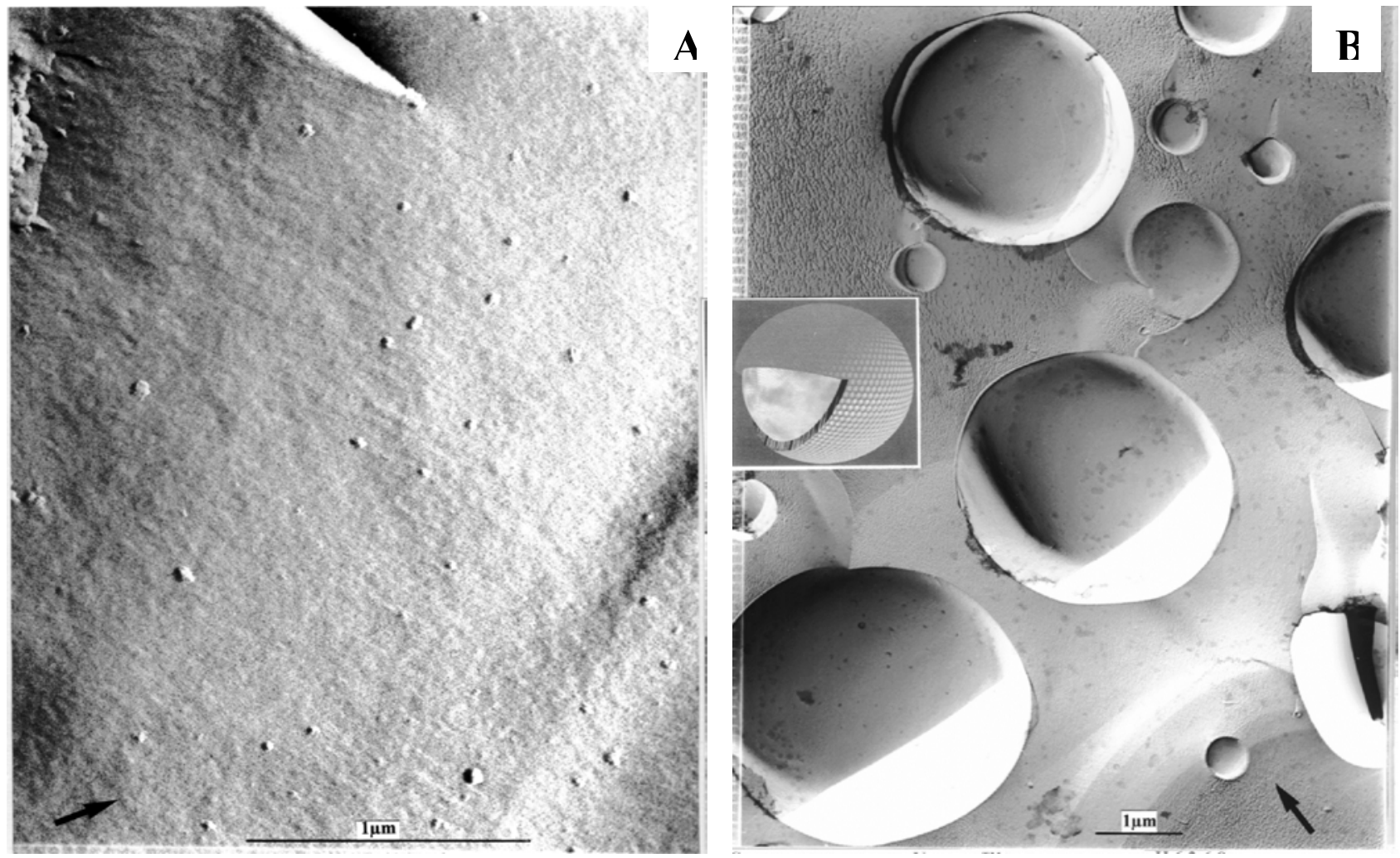

Fig. 1. Freeze-fracture electron micrographs of the ultrasound contrast enhancer Definity®. A.

Sample as received. B. Sample activated by Vialmix ${ }^{\text {TM }}$. C. Sample $250 \mathrm{x}$ diluted. Scale bars $=1 \mu \mathrm{m}$. The shadow ddirection is marked by an arrow.
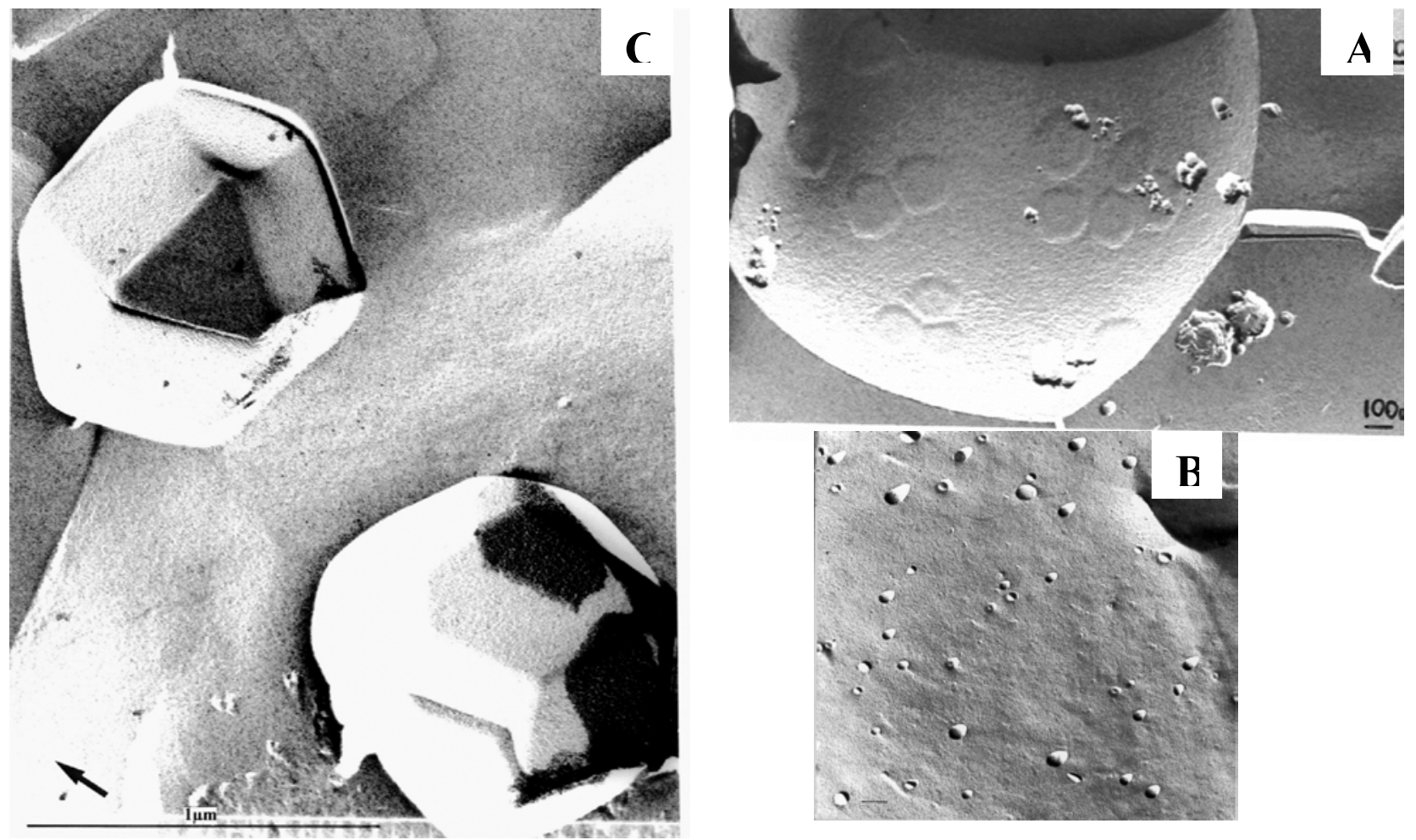

Fig. 2. Freeze-fracture electron micrographs of A. Oil domains in lipid monolayer-stabilizing gas bubble and of B. Small bilayer vesicles (SUV). Scale bars $=100 \mathrm{~nm}$. The shadow direction is running from bottom to top. 